\title{
Incremental profile ring rolling with axial and circumferential constraints
}

\author{
Christopher Cleaver, Julian Allwood (1) \\ Department of Engineering, University of Cambridge, CB2 1PZ; Submitted by Prof. Julian Allwood
}

If profile ring rolling could be achieved without part-specific tooling, significant savings in material, energy and downstream processing could be realised. One approach, 'incremental ring rolling' previously suffered difficulties controlling material flow, resulting in multiple form errors. Inspired by studying an expert using a potter's wheel, two additions to this process are proposed; the use of axial and circumferential constraints. A 12-axis ring rolling machine has been built to demonstrate these process enhancements, producing metal rings up to $1 \mathrm{~m}$ in diameter. The production of both rectangular and L-shape rings is examined, showing significant improvements in ring cross-sectional form and circularity.

Rolling, Flexibility, Ring rolling

\section{Introduction}

Rolled rings are used in annular components in aerospace, oil and gas, electricity generation and other industrial sectors, often with shaped, or 'profiled', cross-sections (Figure 1a).

In the typical ring rolling process, a pierced preform prepared by open-die forging is rolled in a machine between two pairs of tools acting on the radial and axial surfaces of the ring. Currently the only way to produce profiled rings such as railway wheels or aerospace casings is to use shaped tooling (Figure 1b). This is costly; the shape of the preform must be designed, shaped tooling for rolling (often multistage) and forging must be produced. Frequently, rectangular cross-section rings are produced instead and the final shape is cut away (Figure 1c). The aim of this work is to create profiled rings without part-specific tooling to either reduce tooling costs or save material and other inputs.

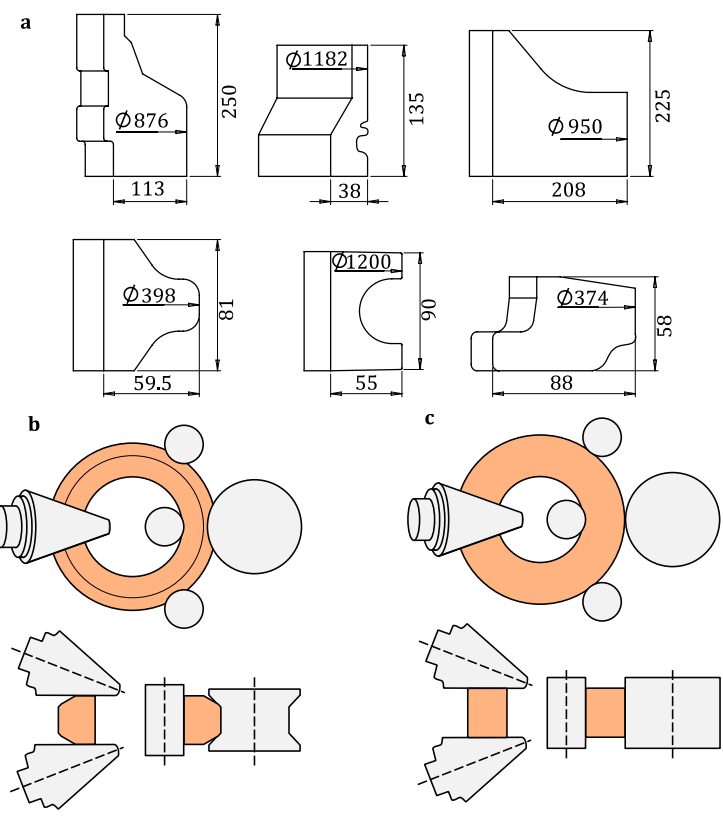

Figure 1 a. Profile ring half sections from Marczinski in mm [1] b. profile ring rolling to produce shaped ring $\mathbf{c}$. plain ring rolling
Incremental profile ring rolling (IRR) was previously proposed as a way to produce profiled rings with a single tool-set albeit with an initially more complex machine [2]. To generate an inner profile by IRR a short vertically moving inner radial tool is used to reduce the wall thickness of the ring by different amounts along its axial height (Figure 2a.) Equivalently, an outer profile shape could be generated with a short outer radial tool.

IRR was studied at IBF, RWTH Aachen, Germany, firstly on an experimental wax ring rolling machine [3]. The results in Figure $2 b$ show that whilst it is possible to create a difference in ring thickness along the height, the resulting rings suffer from conicity and dishing defects driven by non-uniform circumferential extension. Similar results were obtained on trials on cold-rolled steel rings carried out at ORS Bearings, Turkey, as shown in Figure 2c [2]. These defects are so large that there is little or no material saving and to date the incremental ring rolling process has not been taken up.
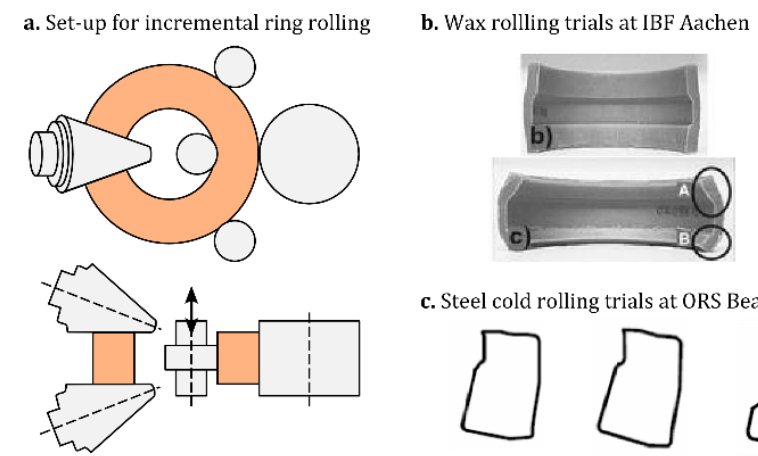

c. Steel cold rolling trials at ORS Bearings
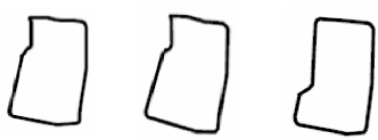

Figure 2. a. Set up for incremental ring rolling, b. results of trials in wax [3] c. results of trials in bearing steel [2]

\section{Concepts for increased flow control in IRR}

To overcome these difficulties increased control over material flow seems necessary; two ways to achieve this are discussed.

One way is to achieve shaping by axial displacement of material, leading to an increase in workpiece height. This is similar to both axial profile tube forming in which a short tool acts on a large tubular workpiece which restricts circumferential flow and also 
pottery throwing, in which the workpiece rotates on a potter's wheel and a craftsperson 'lifts' and shapes the side walls.

Numerous trials were carried out on the University of Cambridge model rolling machine - machine described in [4] - using modelling clay. Two novel set-ups are highlighted here: the first uses a set of constraining rolls around the ring added to the basic incremental ring rolling set-up - Fig $3 \mathrm{~b}$. The second is like the potter's wheel; the ring is fixed at its base onto a rotating platen and passes between radial tools as shown in Figure 3c.

The effect of these constraining features was studied on the creation of many ring shapes; three key results are shown in Fig 3. In each, an L-shape ring is made by reducing the upper region wall thickness by $40 \%$ whilst the lower region was undeformed. With the conventional unconstrained set-up the as-measured ring cross-section shows significant conicity and dishing but with both the additional circumferential constraints and the potter's wheel set-up, axial flow is greater and the conicity is much reduced.
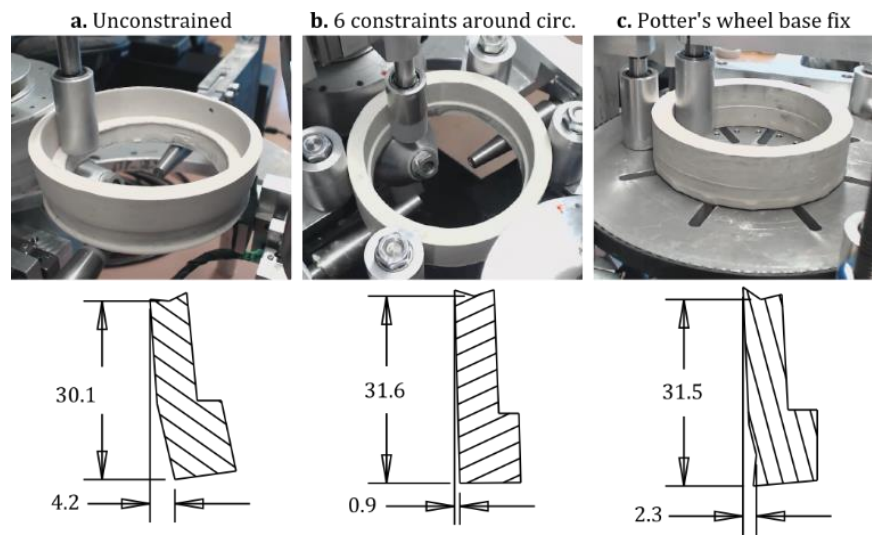

Figure 3. Shaping by increasing the ring height: preliminary clay trials

A second shaping method is to create a change in thickness whilst the ring diameter also increases. In this, for compatibility, internal axial material flow is required from the thinnest into the thickest regions. Previous work suggested that such flow can be enhanced by using a 'closed' radial pass [5].

A novel set-up to achieve this was implemented on the model machine by using a pair of cylindrical axial rolls (Fig $4 \mathrm{~b}$ ). This was trialled on the production of an L-shape ring in which both the upper region was reduced in thickness by $50 \%$ and the lower region was reduced in thickness but by only $25 \%$. The results suggest that the novel set-up can enhance internal axial flow: a difference in thickness is achieved whilst the ring increases in diameter by $20 \%$.
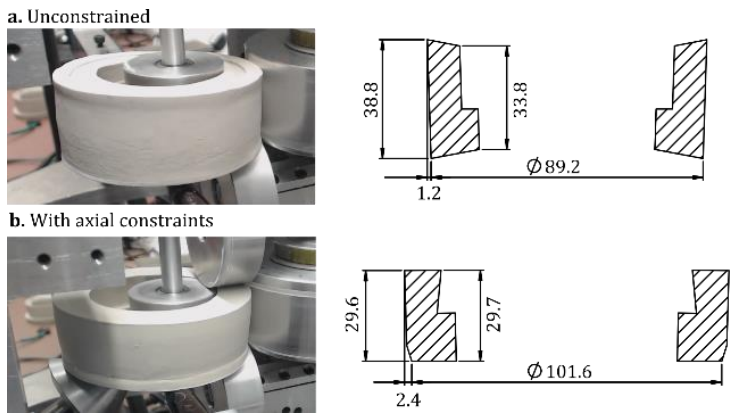

Figure 4. Shaping whilst increasing the diameter: preliminary clay trials

These physical trials on a model clay material give confidence about the kind of additional tooling that might be required to achieve predictable ring forms in incremental ring rolling.

\section{New process and equipment for incremental ring rolling}

To investigate a selection of these concepts on an industrially relevant scale and material, new equipment was commissioned.

The chosen tool arrangement combines the axial and circumferential constraint concepts. There are four overlapping working tools in a novel 'combined radial and axial stage' and six constraint rolls around the ring circumference. Plain radial-axial ring rolling (Fig. 5a) can be achieved by rotating the outer radial tool and reducing the radial and axial roll gaps. Inner and outer incremental rolling (e.g. Fig 5b) is enabled by vertical motion of the inner and outer tool respectively.

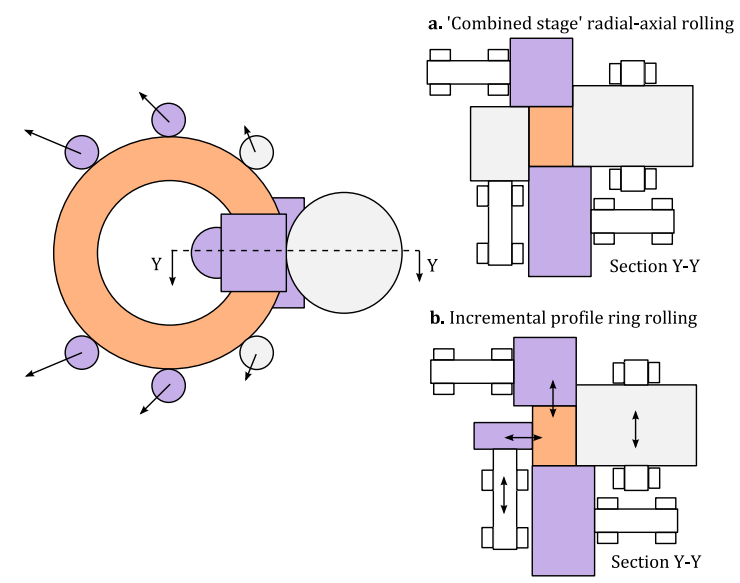

Figure 5. Concept for ring rolling with flexible axial and circumferential constraints a. plain ring rolling, b. inner incremental profile rolling [6]

The 12-axis machine was designed and commissioned as shown in Figure 6 with radial force and axial force capacity of $55 \mathrm{kN}$ and $45 \mathrm{kN}$ respectively, and maximum main roll torque of $1800 \mathrm{Nm}$. This was estimated to be sufficient to process lead rings with maximum wall thickness and height 52 and $78 \mathrm{~mm}$, and pure aluminium (1050-H6) rings up to 21 and $32 \mathrm{~mm}$, as justified next.

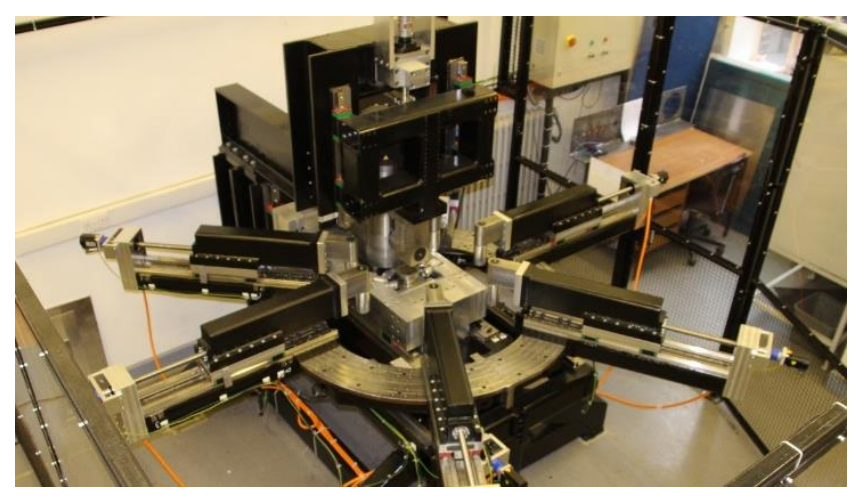

Figure 6. Constrained incremental rolling research machine

\subsection{Force and torque in 'combined stage' radial-axial rolling}

The four-roll 'combined stage' arrangement is unique and its effect on roll force and torques was unknown. As a brief aside, the analytical estimate used for sizing the machine is now compared to experimental results on lead rings.

The radial roll force, $F_{r}$, axial roll force, $F_{a x}$, and outer radial roll torque, $Q$, in 'combined stage' radial-axial rolling were estimated by Equations 1-3, adapted from Hawkyard et al. [7]:

$$
\begin{aligned}
& F_{r}=l_{a v}^{r} H \bar{\sigma} \gamma_{r} \\
& F_{a x}=l_{a v}^{a x} T \bar{\sigma} \gamma_{r} \\
& Q=\frac{V R_{F}}{2 \pi R} \bar{\sigma} \gamma_{r}\left(\varepsilon_{r}+\varepsilon_{a x}\right)
\end{aligned}
$$


where $l_{a v}^{r}$ and $l_{a v}^{a x}$ are the average radial and axial roll contact lengths respectively following the assumptions in [7], $H$ is the ring height, $T$ is the wall thickness, $V$ is the ring volume, $R_{F}$ is the forming roll radius, $R$ is the ring outer radius, $\bar{\sigma}$ is the flow stress, $\varepsilon_{r}$ and $\varepsilon_{a x}$ are the $\gamma_{r}$ is the pressure factor, from slipline field theory, for plane strain indentation of a workpiece of thickness $T$ between equal indenters of width $l_{a v}^{r}$

The ring was reduced in thickness and height by $28 \%$ and $14 \%$ respectively, as detailed in Table 1 . The predicted and observed roll torque and force are compared in Figure 7. Although it captures the trend well, the analytical model under-predicts the roll forces by up to $30-40 \%$, probably because of the interaction between the two rolling stages. The measured torque values are implied from the motor current, possibly explaining the early differences. However, the accuracy is sufficient to justify the machine sizing (which included a factor of safety) and the forces are expected to be smaller during the IRR process itself.
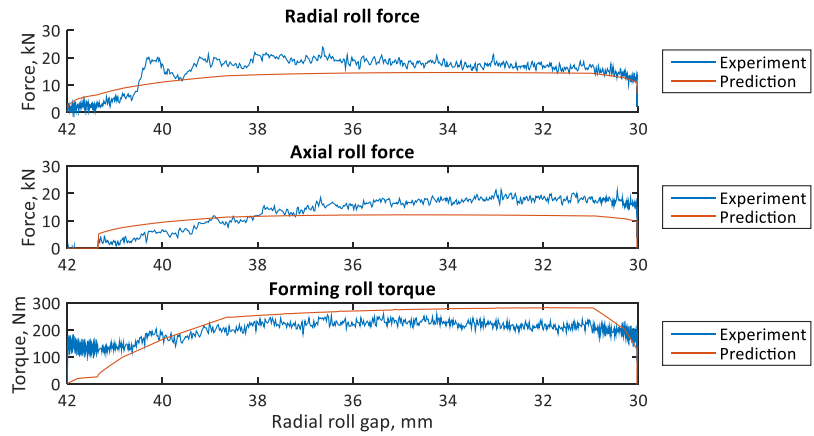

Figure 7. Comparison of analytical, FEM prediction and observed force and torque for 'combined stage' radial-axial rolling of lead BS EN 12588.

\section{Evaluation of new process on production of L-section rings}

Selected results from the first trials on the innovative incremental rolling processes are now shown on the production of L-section rings previously unachievable by IRR.

\subsection{Methodology}

The targeted L-shape cross-section is shown alongside the rectangular cross-section pre-rolled preform in Fig 8a-b. To assess the effect of the new constraints, three trials were carried out with each added in turn, as shown in Figure 8c-e. When the circumferential constraints were used, the leading constraint roll was set to apply a force of approximately $2 \mathrm{kN}$, the remaining rolls controlled to have the same radial position. When the axial rolls are used they are set to maintain a constant height.
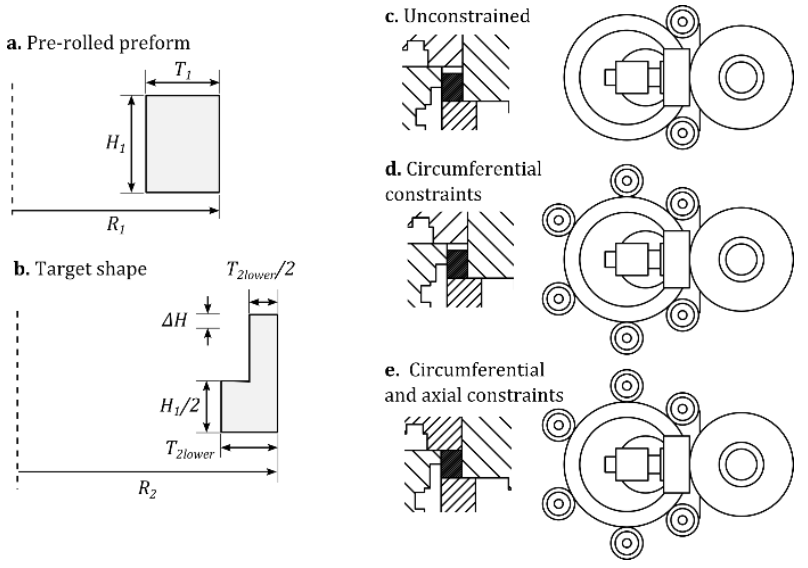

Figure 8. a-b. Preform and target shape, c-e process set-ups
To create the L-shape profile, the upper region was first reduced in thickness by a nominal amount $(10.5 \%)$ over 5 rotations. This results in a ring of conical form as shown in Figure $9 \mathrm{~b}$. To correct this, the lower section is then reduced in thickness until the upper radius is equal to the lower radius (Fig 9d) as measured by a laser profile scanner. The process was repeated for sufficient number of iterations until the ratio $\Delta$ of the upper thickness to the lower thickness is less than 0.5 .

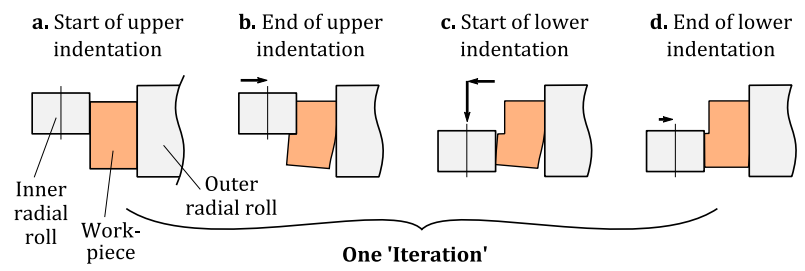

Figure 9 Incremental shaping tool movement

Before the shaping operation, the cast lead preforms were rolled to a specified thickness and radius under conditions as in Table 1. This was carried out achieving the tolerances reported in the table.

Table 1 Parameters in the production of L-shape rings

\begin{tabular}{|l|r|r|}
\hline Parameter & Pre-roll & \multicolumn{1}{l|}{ Shaping } \\
\hline Forming roll radius & $100 \mathrm{~mm}$ & $100 \mathrm{~mm}$ \\
Mandrel radius & $45 \mathrm{~mm}$ & $55 \mathrm{~mm}$ \\
Forming roll speed & $0.2 \mathrm{rev} / \mathrm{s}$ & $0.2 \mathrm{rev} / \mathrm{s}$ \\
Thickness reduction (per rev.) & $4 \%$ & $3 \%$ \\
Height reduction (per rev.) & $2 \%$ & $\mathrm{~N} / \mathrm{A}$ \\
Initial workpiece radius, R & $98.0 \mathrm{~mm}$ & $135.0 \mp 0.10 \mathrm{~mm}$ \\
Initial workpiece wall thickness, $\mathrm{T}$ & $41.5 \mathrm{~mm}$ & $30.2 \mp 0.02 \mathrm{~mm}$ \\
Initial workpiece height, H & $46.5 \mathrm{~mm}$ & $40.8 \mp 0.02 \mathrm{~mm}$ \\
\hline
\end{tabular}

The stress-strain response of the lead material was evaluated in a tensile test. The response is similar to hot forming aluminium or steel: lead recrystallizes at room temperature and there is both strain and strain-rate hardening. For predicting forces in Section 3 where the strain rate is approximately $0.2 / \mathrm{s}$, the Holloman equation $\left(\bar{\sigma}=K \bar{\varepsilon}^{n}\right)$ with $\mathrm{K}=35 \mathrm{MPa}$ and $\mathrm{n}=0.26$ was used.

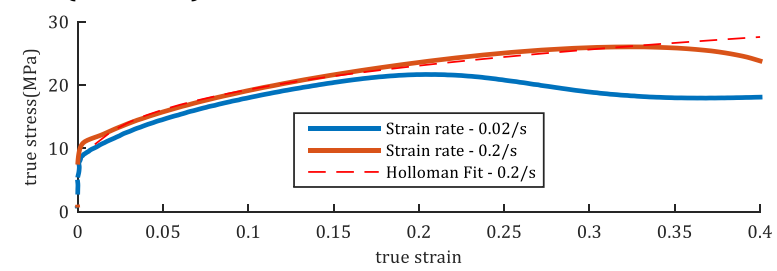

Figure 10. Stress-stain response of lead BS EN 12588 in a tensile test

To investigate the ring stress state an isothermal FEM simulation of the unconstrained upper indentation was carried out using the ABAQUS Explicit solver. Mass scaling of 200 was used. The ring was modelled as a deformable body with $400 \times 20 \times 5=48,000$ brick elements (circumference $\mathrm{x}$ height $\mathrm{x}$ wall thickness). The tools were modelled as rigid bodies. Contact pairs were used with Coulomb friction between the workpiece and outer radial tool, and all other interactions were frictionless. Tabulated flow stress data from Fig. 10 was used.

\subsection{Results}

The shaped rings and cross-sections are shown in Figure 11. The unconstrained ring requires the largest corrective lower strain (36\% of the upper strain) and so the final ring has a larger diameter and reduced thickness. Despite this, the conicity defect is not fully corrected in the lower outer corner. The circumferentially 
constrained ring grows noticeably in height, the final diameter is smaller and the cross-section appears more uniform. The least corrective strain (23\%) is required for the axial and circumferential constraints ring.
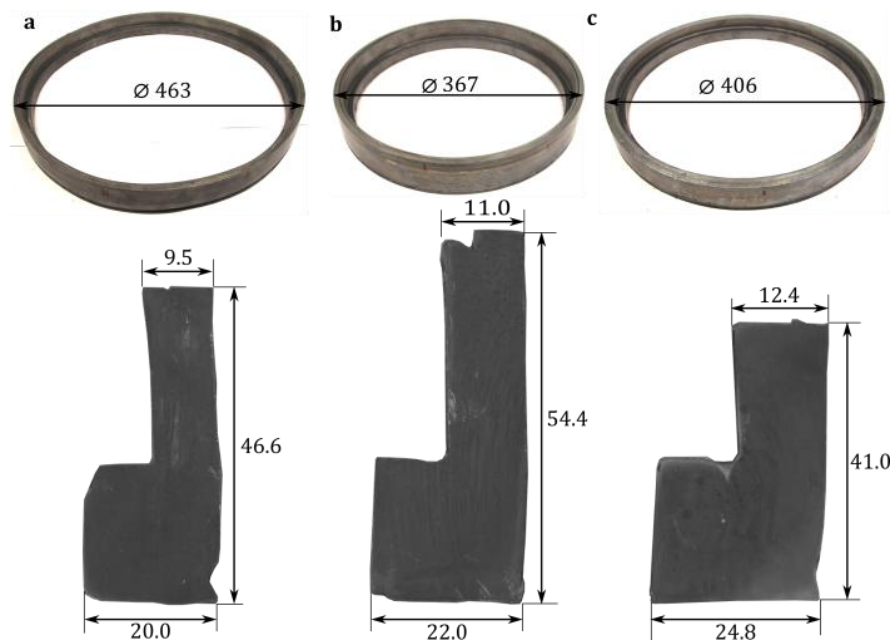

Figure 11. Results of novel incremental ring rolling process to create an Lshape ring in lead (dimensions in $\mathrm{mm}$ ) a. unconstrained b. with circumferential constraints $\mathbf{c}$. with axial and circumferential constraints.

Figure 12 presents three figures of merit for the accuracy of the processes. Firstly, the shaping loss: the ratio of the area that would be lost to machine each face back to square, avoiding flaws, and leaving a ring of step depth, $\Delta, 0.5$. Secondly the material saving: comparing the material used to the material to make a plain ring encompassing the useful area. Finally, the circularity error: the range in as-measured diameters around the ring to the average diameter. All three parameters are much improved by the new constraints: the material saving - negative in the unconstrained ring since so much material would be lost - increases to $22 \%$.

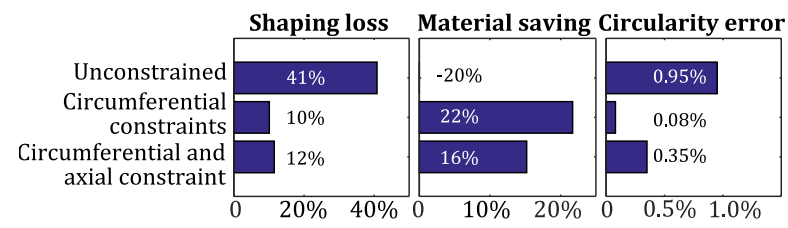

Figure 12. Effect of constraints on process accuracy and effectiveness

The microstructure of the three rings was compared to the rolled preform. An important feature in the unconstrained ring is a crack in the corner region that extends almost $3 \mathrm{~mm}$ into the workpiece. In comparison, in the circumferentially constrained ring, there is a much smaller crack of approx. $0.3 \mathrm{~mm}$ depth. If both constraints are used, the crack is not present. This might be caused by tensile stresses in the lower region when the upper section is being deformed, as predicted by the FEM - see Fig. 13.

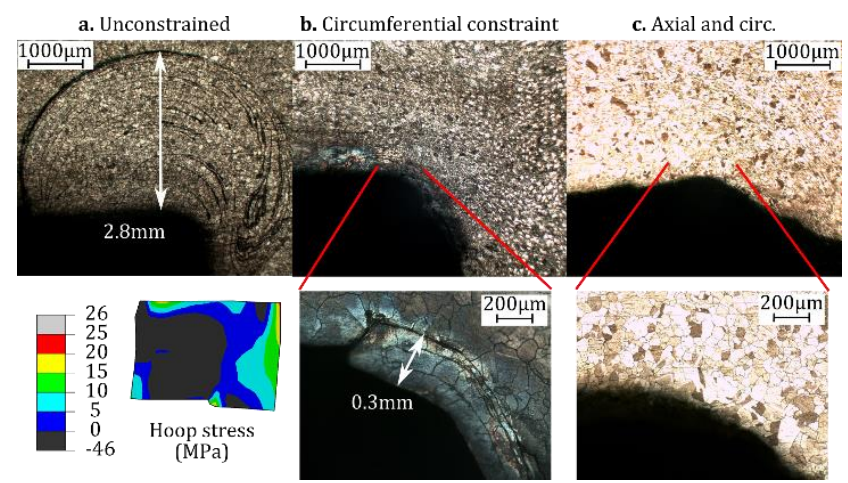

Figure 13. Cracking in the corner region in IRR: effect of constraints

\section{Analysis and discussion}

Having shown a specific size of L-section ring can be made with the new tooling, the application of the process to a wider range of rings of differing step depth, $\Delta$, and ratio of inner radius to wall thickness, $\hat{R}$ is now considered.

Firstly, consider the unconstrained process, as shown in Fig. 14. The results suggest at least three limiting factors: the ring cracked, suggesting a limit the step depth, $\Delta$, perhaps to $75 \%$ of the value in the experiment as is drawn. The ring circularity is too poor to be useful: this might be governed by the ratio of ring radius to its plastic section modulus: a second line is drawn at $75 \%$ of the value in the experiment. Finally, the shaping operation requires a secondary strain, so there is a limit to how thick-walled the ring can be without requiring a preform with unrealistically small hole. A third 'preform limit' line is plotted assuming the secondary strain and height growth is unchanged and a minimum initial $\widehat{\mathrm{R}}=1.0$.

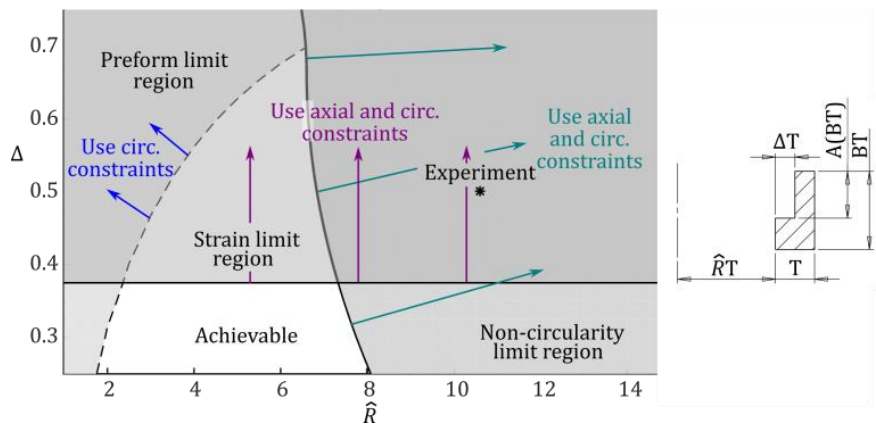

Figure 14. Estimated operating window for L-section rings of varying step depth, $\Delta$ and radius $\hat{R}$ by unconstrained IRR, showing effect of constraints

The use of the axial and circumferential constraints is expected to increase the operating window: both together eliminated cracking in the experiment (with $\Delta=0.5$ ) and produced a ring with acceptable circularity. Therefore, their use is recommended in the areas shown by arrows on Fig 14. Also, it appears using the circumferential constraints alone makes it possible to produce thicker walled rings: the ring grows in height instead of diameter.

Overall, the results and analysis show that material savings can be achieved by the new constrained Incremental Ring Rolling process, without requiring part-specific tooling. A comparison of the ring cross-sections in Figure $11 \mathrm{~b}-\mathrm{c}$ to Figure 2 obtained in past work, shows that a significant step forward has been made. Further exploration of the process limits, especially for larger and small diameter shaped rings, as estimated in Figure 14, is recommended alongside investigation of other industrially relevant shapes and upscaling to 'real' engineering materials.

\section{Acknowledgements}

The first author was supported by an EPSRC I-Case Studentship (12220703), with Primetals Technologies Ltd. Prof. Allwood was supported by EPSRC grant EP/K018108/1.

\section{References}

[1] Marczinski, H. J. (1984). The Hot Ring Rolling Process and its Integration into Automatic Production Lines. Proc. 3rd Int. Conf. on Rotary Metalwork, 251-265

[2] Allwood, J. M., Kopp, R., Michels, D., Music, O., Öztop, M., Stanistreet, T. F., Tekkaya, A. E., Tiedemann, I. (2005). The Technical and Commercial Potential of an Incremental Ring Rolling Process. CIRP Annals - Manufacturing Technology 54(1), 233-236.

[3] Tiedemann, I., Hirt, G., Kopp, R., Michl, D., Khanjari, N. (2007). Material flow determination for radial flexible profile ring rolling. Production Engineering, 1(3), 227-232. [4] Stanistreet, T. F., Allwood, J. M., Willoughby, A. M. (2006). The design of a flexible model ring rolling machine. Journal of Materials Processing Technology, 177(1-3), 630-633.

[5] Moussa, G. (1981). An investigation into the rolling of profiled rings. PhD Thesis.

[6] Allwood, J. M., Cleaver, C. J. (2016) Ring rolling process and apparatus for ring rolling. Patent Application. Pub. No.: WO/2016/142661

[7] Hawkyard, J. B., Johnson, W., Kirkland, J., Appleton, E.. (1973). Analyses for Roll Force and Torque in Ring Rolling, with some Supporting Experiments. Int. J. Mech. Sci., $15,873-893$. 